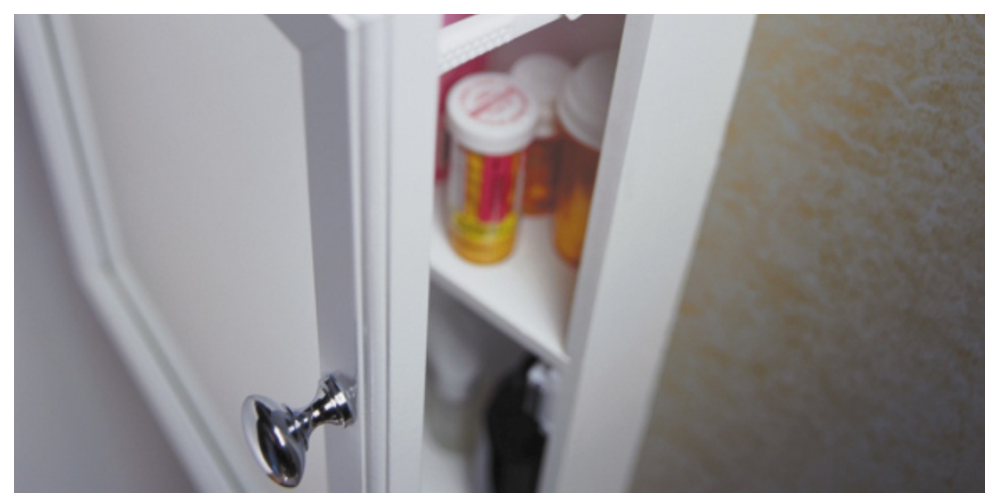

APOPTOSIS

\section{Key to the poison cabinet}

Toxic factors such as cytochrome $c$ are locked deep within the mitochondria. In response to certain death-promoting stimuli, however, they can be released into the cytosol. The key to this cellular poison cabinet is not known, but a report in Science by Craig Thompson, Stanley Korsmeyer and colleagues now points to the pro-apoptotic proteins Bax and Bak.

Previous studies had already implicated these proteins in the release of cytochrome $c$ from mitochondria. Activation of death receptors leads to the cleavage of a protein called Bid to its truncated, active form, $\mathrm{tBid}$. This, in turn, induces the homooligomerization of Bax and Bak, leading to cytochrome $c$ release.

To investigate whether Bax and Bak are required for tBidinduced apoptosis of whole cells, the authors used a retroviral vector to express tBid in mouse embryonic fibroblasts (MEFs). Whereas wild-type, Bak-deficient and Bax-deficient MEFs showed a shrunken, apoptotic morphology, the Bax, Bak doubledeficient MEFs did not die.

One explanation is that $\mathrm{tBid}$-induced apoptosis operates through the activation of Bax and Bak. To test this possibility, Thompson, Korsmeyer and colleagues asked whether these proteins are activated in the tBid-expressing MEFs, and they observed Bax and Bak oligomers - indicative of active protein - in the presence of tBid. Also in support of this idea, in the Bax, $B a k$-deficient MEFs there was no detectable redistribution of cytochrome $c$ from the mitochondria to the cytosol.

The authors next asked whether Bax and Bak are required for cell death in situ by assessing apoptosis in mouse livers. Again, they found that tBid-dependent apoptosis requires Bax or Bak. The death-promoting stimulus used in these experiments was activation of the death receptor Fas, but the authors confirmed that Bax and Bak are also required for death in response to a host of other factors, including stress signalling from the endoplasmic reticulum.

The authors conclude, then, that the "activation of a 'multidomain' proapoptotic member, Bax or Bak, appears to be an essential gateway to mitochondrial dysfunction required for cell death in response to diverse stimuli".

(2) References and links

ORIGINAL RESEARCH PAPER Wei, M. C. et al. Proapototic BAX and BAK: A requisite gateway to mitochondrial dysfunction and death. Science 292, 727-730 (2001) FURTHER READING Finkel, E. The mitochondrion: is it central to apoptosis? Science 292 FURTHER REA

\title{
Wingless but moving
}

Proteins that act at specific cellular locations not only need to be synthesized at the right time but they also need to be transported to the right place. Two solutions exist to this geographical problem: either the proteins are synthesized throughout the cell and then transported to the right location, or the messenger RNA (mRNA) is transported to the right location, where the protein is then translated. The latter approach is less well understood than the former, and is the object of two studies reported in Cell.

Many components of the wingless signalling pathway, including wingless itself, localize apically in polarized cells. Simmonds and colleagues used a sensitive fluorescen in situ hybridization technique and confocal microscopy to study the localization of wingless transcripts in the ectoderm of stage 8 Drosophila melanogaster embryos. They defined two regions in the 3' untranslated region (UTR) of the mRNA of wing less that act in concert to localize the RNA apically. Using transgenic flies with mutated 3' UTRs, the authors showed that the apical localization of wingless $\mathrm{mRNA}$ and wingless protein is required for signalling through the wingless pathway (see picture).

So, how is the wingless mRNA transported to the apical pole of cells? Wilkie and Davis studied the localization of several apically targeted transcripts in Drosophila syncytial blastoderm embryos, in which thousands of nuclei are aligned in a monolayer and the cytoplasm surrounding them is highly polarized. The authors injected AlexaFluor dye-labelled mRNAs into the basal cytoplasm of the blastoderm embryo, and followed their transport to the apical pole. They found that the mRNAs of wingless and of the pair-rule transcripts runt and fushi tarazu were rapidly incorporated into transport particles and exported out of the nucleus in a non-directional manner. Although different mRNAs were transported in one particle, each transport particle probably contained only a few mRNA molecules. Transport to the apical pole of the blastoderm embryo was rapid and directed, and depended on microtubules, the molecular motor dynein, and the linker protein complex dynactin.

The two studies are complementary, one demonstrating the functional importance of the apical localization of the wingless transcript, and the other presenting a possible mechanism through which this localization is achieved. Although each of the two studies considered only one cell system, similar mechanisms probably operate in many cells in which mRNAs are localized. Many questions remain: what holds together the transport particle? What are the linker molecules connecting the particle and the molecular motor? And, how do these linker molecules interact with dynactin?

$$
\text { Raluca Gagescu }
$$

\section{(2) References and links} ORIGINAL RESEARCH PAPERS Simmonds, A. $J$. et al. Apical localization of wingless transcripts is required for wingless signaling. Cell 105, 197-207 (2001) | Wilkie, G. S. \& Davis, I. Drosophila wingless and pair-rule transcripts localize apically by dynein-mediated transport of RNA particles. Cell 105, 209-219 (2001)

FURTHER READING Jansen, R. P. mRNA localization: message on the move. Nature Rev. Mol. Cell Biol. 2, 247-256 (2001)
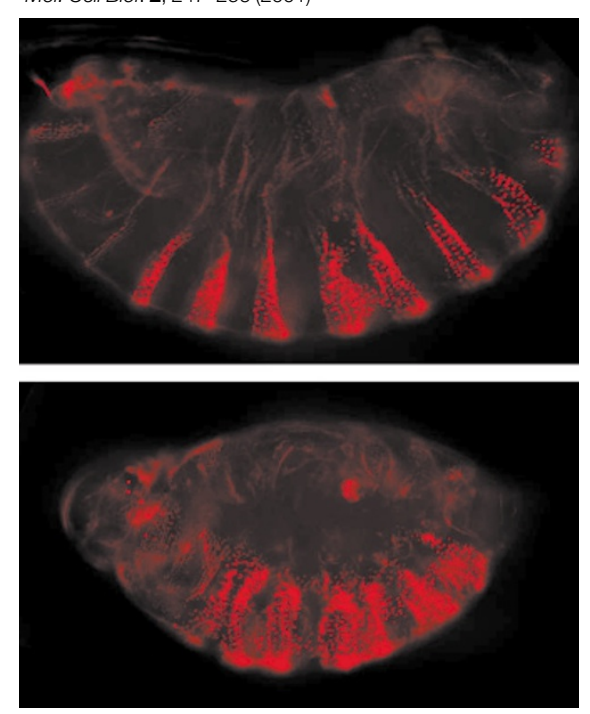

Apically (top) but not basally (bottom) targeted wingless mRNA rescues cuticle formation in Drosophila embryos. Courtesy of A. J. Simmonds, University of Toronto. 